\title{
Evaluation of the structures size in the liquid-gas flow by gamma-ray absorption
}

\author{
Marcin Zych ${ }^{1,}$, , Robert Hanus $^{2}$, Marek Jaszczur ${ }^{3}$, Dariusz Świsulski ${ }^{4}$, Leszek Petryka ${ }^{5}$, Paweł Jodłowski ${ }^{5}$ and Piotr Zych ${ }^{6}$ \\ ${ }^{1}$ AGH University Science and Technology, Faculty of Geology, Geophysics and Environmental Protection, 30-059 Kraków, Poland \\ ${ }^{2}$ Rzeszów University of Technology, Faculty of Electrical and Computer Engineering, 35-959 Rzeszów, Poland \\ ${ }^{3}$ AGH University Science and Technology, Faculty of Energy and Fuels, 30-059 Kraków, Poland \\ ${ }^{4}$ Gdańsk University of Technology, Faculty of Electrical and Control Engineering, 80-233 Gdańsk, Poland \\ ${ }^{5}$ AGH University of Science and Technology, Faculty of Physics and Applied Computer Science, 30-059 Kraków, Poland \\ ${ }^{6}$ Warsaw University of Technology, The Institute of Telecommunications, 00-661 Warszawa, Poland
}

\begin{abstract}
The rapid development of tomography methods particularly electrical, $\mathrm{X}$ and gamma rays allows for a wide range of the information about flow structure. However, all of such methods are quite complicated. At the same time much simpler systems as the measuring system of gamma rays absorption, allows to obtain a all key flow information which describe the two-phase flow. In the article the results of analyzes of radiometric signal that not only allow to recognize the type of flow, but also the assessment of forming structures are presented. Calculation and interpretation of the data were based on the crosscorrelation and cross-spectral density function. In order to verify the calculations the photographic documentation made during the measurements was used.
\end{abstract}

\section{Introduction}

Depending on the velocity of the two-phase mixture of gas-liquid in a pipe and the void fraction $\alpha$, one can obtain different flow structures. The recognition of transport method of the gas phase and the assessment of the size of the resulting structures are being increasingly undertaken as research topics. The most often used in such studies are Tomographic methods: electric, X-rays and gamma rays and optical methods [1-5]. Tomography systems require the installation of sensors around the selected sections of the pipeline and from the obtained signals to reconstruct the image showing the distribution of individual gas bubbles, and also outline the basic flow parameters. However, the authors believe that in the case of small diameter pipelines one can use a simpler measurement systems that also allow the assessment of the flow characteristics, the type of identification and estimation of the size of the resulting structures.

With a small diameter pipe (less than $50 \mathrm{~mm}$ ), the flow system as a pipeline is often reduced to the twodimensional problem, where it is sufficient to know the length and depth of the resulting structures.

The measuring system proposed in this article is a set of measurement devices based on the absorption of gamma radiation, which is being perfected by the authors for many years [6-8].

\section{Laboratory stand}

The hydraulic system built in the Sedimentological Laboratory of the Faculty of Geology, Geophysics and Environmental Protection, AGH in Krakow was used for measurements. A fragment of the experimental system is shown in Fig. 1.

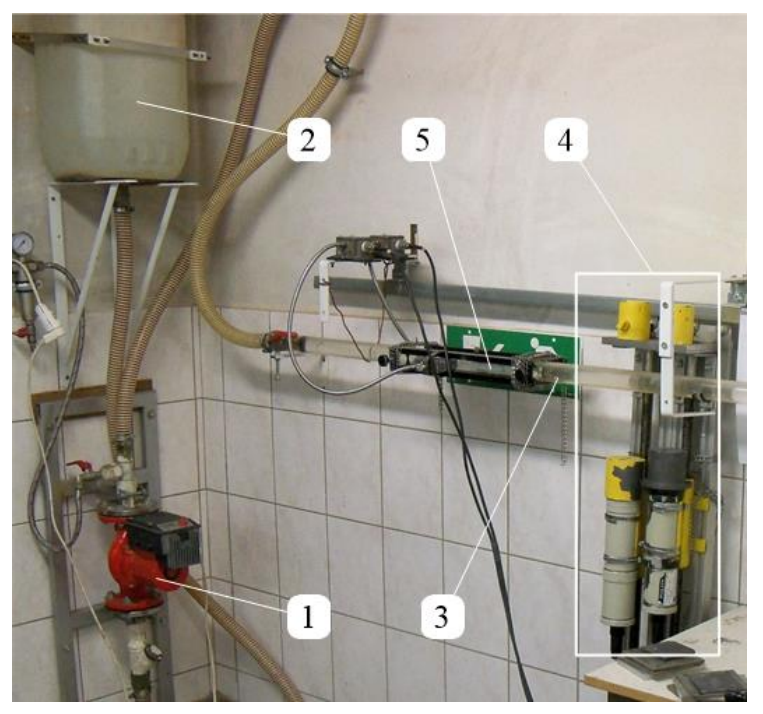

Fig. 1. Fragment of laboratory stand for the measurement of two-phase liquid-gas flow: 1 - pump, 2 - expansion tank, 3 - pipeline, 4 - gamma absorption measurement system, 5 - ultrasonic flowmeter. 
A set for measuring the absorption of gamma radiation consists of two scintillation probes and a gamma-ray Am-241 sources of about $100 \mathrm{mCi}$ activity. The pipe through which flows the water-air mixture is formed with a horizontal pipe made of metapleks with a length of $4.50 \mathrm{~m}$ and an internal diameter of $30 \mathrm{~mm}$. The flow velocity of the liquid is contained in the range of $0.17-3.60 \mathrm{~m} / \mathrm{s}$, allowing to obtain the following types of movement: stratified (wavy), slug, plug and bubble flow.

The measuring stand, in addition to the absorption of gamma radiation and ultrasonic flowmeter, is equipped with a camera for independent tracking of the movements of the gas phase.

The laboratory stand along with a description of the measurement system for the absorption of gamma radiation has been widely described in articles $[9,10]$.

\section{Analysis of time and frequency signals}

In the experiments carried out, stochastic signals were obtained, which are characterized by a large proportion of noise. Depending on the amount of the gas and the flow rate of the mixture the signal changes as can be observed in the frequency and time domains [10]. For the presentation of the possibilities of the proposed method, an experiment was chosen for analysis in which the structure of slug flow was achieved. This type of movement arising from the large amount of air supply is characterized by very large blisters of gas filling almost the entire cross-section of the pipe (Fig. 2), and smaller bubbles flowing in the spaces between large structures (Fig. 3). These structures clearly exhibit the time course of signal, a portion of which is shown in Fig. 4.

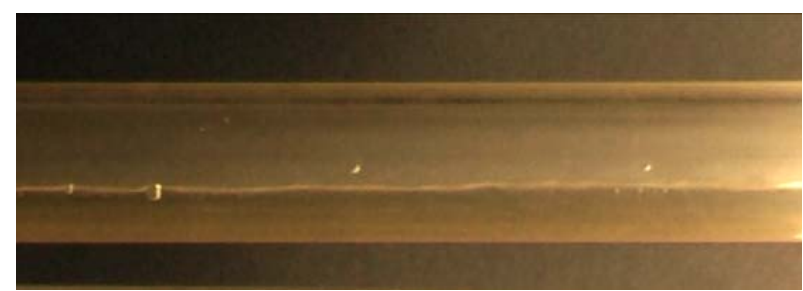

Fig. 2. Fragment of a large bubble entrusts resulting from the slug flow.

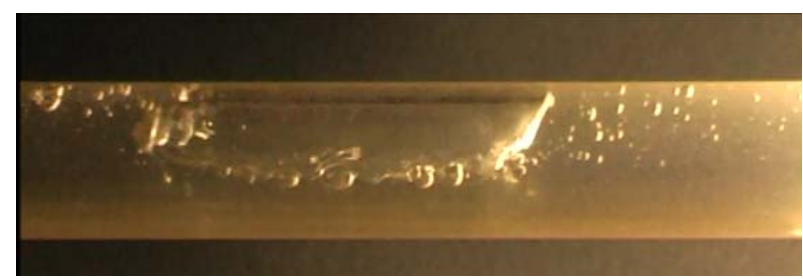

Fig. 3. Small bubble formed in the slug flow.

The experiment analyzed in the article was conducted at an average velocity of water $v_{W}=2.08 \mathrm{~m} / \mathrm{s}$ (measurement done by ultrasonic flowmeter), the average velocity of the air $v_{A}=(1.325 \pm 0.053) \mathrm{m} / \mathrm{s}$ and an average gas void fraction $\alpha=0.434$ [11].

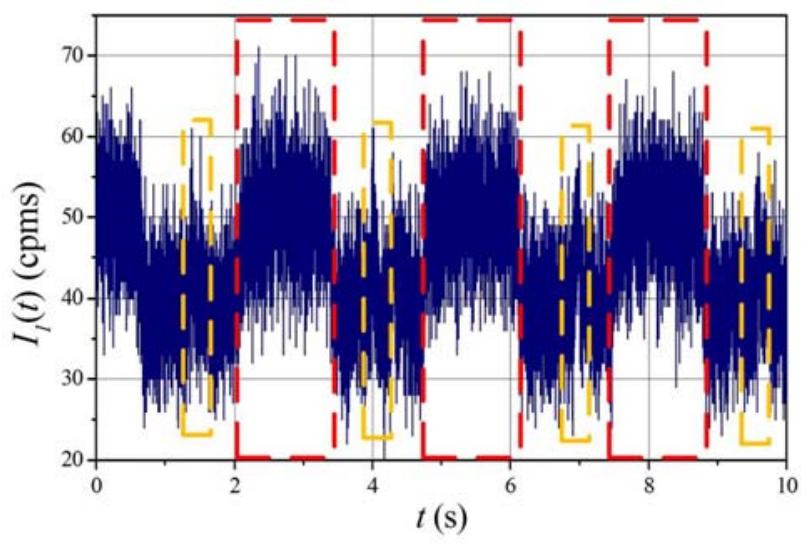

Fig. 4. The first $10 \mathrm{~s}$ of signal registered for the described experiment. Red frame indicates the signal coming from the flow of large air bubbles, and an orange frame from smaller structures.

Using filtering of signal from Fig. 4 one receives smoothed data as shown in Fig. 5.

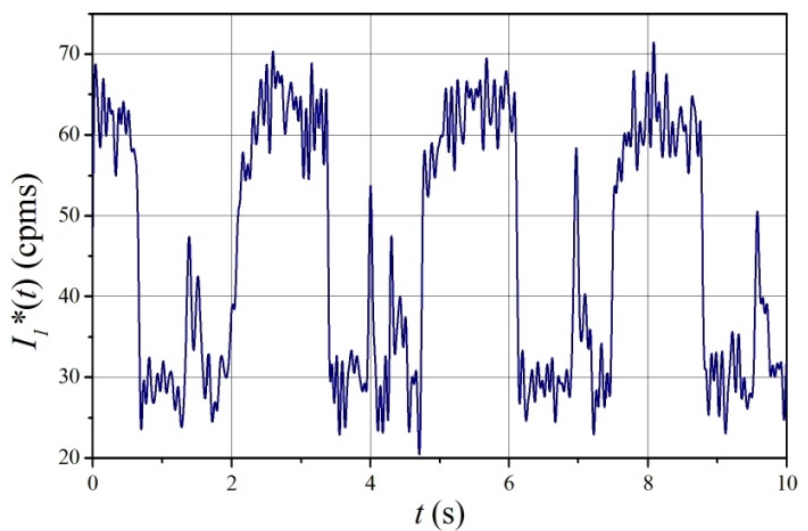

Fig. 5. The first $10 \mathrm{~s}$ of filtered signal registered for the described experiment.

The shown representation of the signal in the time domain shows that it is a superposition of a square wave function associated with the movement of large bubbles of gas, and features related to the movement of small bubbles. Such a course of time should include a frequency characteristic in the frequency domain. For large structures, it will be a distinctive set of frequencies that appear with fallowing ones being odd multiples of the fundamental frequency, according to:

$$
f_{n}=(2 \cdot n+1) \cdot f_{0}
$$

where: $f_{0}$ - fundamental harmonic with the highest amplitude, $f_{n}$ - another characteristic harmonic in the spectrum $n \in\{1,2, \ldots\}$.

For the analysis in the frequency domain a cross spectral density function (CSDF) was used, obtained by the Fourier transform of the cross-correlation function (CCF) $[10,12]$. For reason of clarity, Fig. 6 shows the frequency spectrum with marked characteristic frequencies. The red lines indicate the first six harmonics associated with the movement of large bubbles described by equation (1). In turn, the first 5 harmonics $f_{k}$ marked 
with orange lines are even multiples of dominant frequency $f_{0}$ described by the relationship:

$$
f_{k}=2 \cdot k \cdot f_{0}
$$

for $\mathrm{k} \in\{1,2,3, \ldots\}$.

On the basis of the signal waveforms and photographic documentation it has been identified that these are frequencies related to the movement of smaller structures, located in the spaces between large blisters. The red dashed line, forming a frame indicates a useful range of signal frequencies on the outskirts of the determined cut-off frequency in order to reduce noise [10].

a)

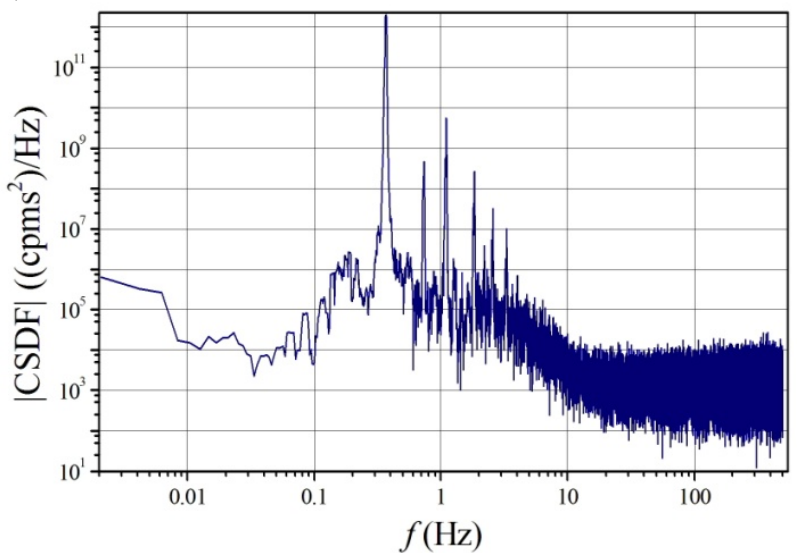

b)

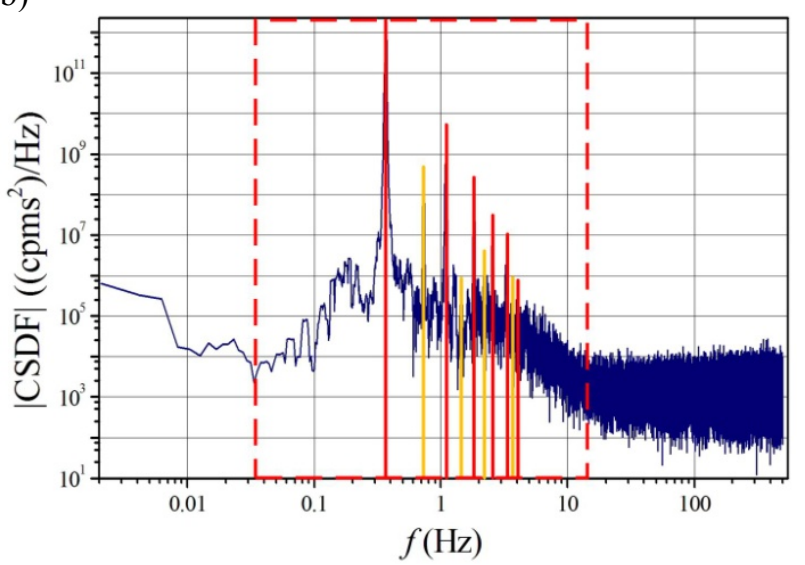

Fig. 6. The spectrum $|\mathrm{CSDF}|$ for slug flow (a); marked characteristic frequencies: red lines - the flow of large bubbles, orange lines - the flow of small bubbles, red frame shows the range of the useful frequencies (b).

Based on the time signal and the knowledge of the flow velocity $v_{A}$ (calculated from the course of the CCF $[13,14])$ of structures formed by the air one can calculate the length $l_{n}$ of individual bubbles from the formula:

$$
l_{n}=t_{n} \cdot v_{A}
$$

where, $t_{n}$ - the flow time of a large bubble of air registered by a scintillation probe. This time is reflected in the data by an important increase in the number of counts.
The second method for determining the length of the largest structures created by the air, is the analysis of the characteristic frequencies of the spectrum $|\mathrm{CSDF}|$. The harmonic which is associated with the movement of the large bubbles is the domain frequency $f_{0}$, which allows one to calculate the average length $l$ of the largest structures in the entire duration of the measurement (480 s), from the relationship:

$$
l=\frac{v_{A}}{2 \cdot f_{0}}
$$

The average size of smaller structures in the flow are associated with even-numbered multiples of $f_{0}$. For the medium bubbles it is for example $f_{10}=10 \cdot f_{0}$.

Another important issue is the depth of the resulting structures, which can be determined based on the absorption of gamma radiation. However, this requires the prior calibration of the measuring system, which is described in the article [11]. The calibration procedure is the same as when determining the void fraction $\alpha$. The method makes it possible to assess the depth of single large bubbles $d_{n}$ from the relationship:

$$
d_{n}=\ln \left(\frac{I_{n}}{I_{0}}\right) / 0.0111
$$

where: $I_{n}$ - intensity of radiation in the interval, where a big bubble flows, $I_{0}$ - intensity of radiation for the pipeline completely filled with water (reference point).

The method also allows the determination of the mean depth $d$ of single large bubbles. However, when calculating the average value one should calculate the coefficient $C$ which is the logarithm of the ration of the number of counts for passages where there were large parts of the structure to the signal, which formed small bubbles. After performing the calculations for a number of cycles of the flow of large and smaller structures, it was found that the value of $C=2$. So finally the average depth $d$ of a big bubble for the entire experiment (i.e. $480 \mathrm{~s}$ ) is given by:

$$
d=2 \cdot \ln \left(\frac{I}{I_{0}}\right) / 0.0111
$$

where: $I-$ the average radiation intensity for the entire measurement.

The uncertainty of the size of the individual values $u_{c}$, which are determined in an indirect way, is based on the law of propagation of uncertainty as recommended by IMEKO [15].

\section{The results of the experiment}

Table 1 shows the current and average length and depth of large bubbles for the first 10 seconds of the flow and the average values for the whole experiment, lasting 480 seconds. 
Table 1. The values of instantaneous and average length $l_{n}$ and depth $d_{n}$ of large bubbles.

\begin{tabular}{|c|c|c|c|c|}
\hline $\begin{array}{c}\text { No. of } \\
\text { large } \\
\text { structure }\end{array}$ & $\begin{array}{c}l_{n} \\
(\mathrm{~m})\end{array}$ & $\begin{array}{c}u_{c}\left(l_{n}\right) \\
(\mathrm{m})\end{array}$ & $\begin{array}{c}d_{n} \\
(\mathrm{~mm})\end{array}$ & $\begin{array}{c}u_{c}\left(d_{n}\right) \\
(\mathrm{mm})\end{array}$ \\
\hline 1 & 1.78 & 0.07 & 23 & 1.0 \\
\hline 2 & 1.84 & 0.07 & 23 & 1.0 \\
\hline 3 & 1.77 & 0.07 & 22 & 1.0 \\
\hline 4 & 1.83 & 0.07 & 23 & 1.0 \\
\hline 5 & 1.84 & 0.07 & 23 & 1.0 \\
\hline 6 & 1.92 & 0.08 & 24 & 1.0 \\
\hline 7 & 1.90 & 0.08 & 22 & 1.0 \\
\hline 8 & 1.95 & 0.08 & 23 & 1.0 \\
\hline 9 & 1.96 & 0.08 & 22 & 1.0 \\
\hline 10 & 1.99 & 0.08 & 22 & 1.0 \\
\hline $\begin{array}{c}\text { average } \\
10 \mathrm{~s}\end{array}$ & 1.88 & 0.08 & 23 & 1.2 \\
\hline $\begin{array}{c}\text { average } \\
480 \mathrm{~s}\end{array}$ & 1.82 & 0.07 & 25 & 0.7 \\
\hline
\end{tabular}

Shown in Table 1 , the length $l_{n}$ of large structures was calculated according to the formula (3) and the depth $d_{n}$ from the formula (5). In contrast, the average length $l$ of a large bubble for the whole measurement time of $480 \mathrm{~s}$ was calculated from the formula (4), and the depth $d$ from equation (6).

Analyzing values summarized in Table 1 it can be seen that the length of the large structures has changed during the first 10 seconds in the range from $1.77 \mathrm{~m}$ to $1.99 \mathrm{~m}$, oscillating around the average value of $1.88 \mathrm{~m}$. This is the value lying in the range of uncertainty for the average for the whole measurement $(480 \mathrm{~s})$ of $1.82 \mathrm{~m}$. The noticeable difference is probably related to the greater variability in length $l_{n}$ of large bubbles in the longer duration of the experiment. Also, analysis of the depth $d_{n}$ and $d$ of various large structures gives similar values. The accuracy of the proposed methods is not high because of uncertainty does not exceed $5 \%$ of the measured values.

\section{Conclusions}

The article presents two ways of determining the size of structures of two-phase gas-liquid flow of slug flow type based on signal analysis of radiometric probes. Both analyses in the time domain and frequency domain show a high similarity of the results within the measurement uncertainty. The results characterize the variability of the length $l_{n}$ and depth $d_{n}$ of large bubbles very well, what was shown by the detailed analysis within the first 10 seconds of the experiment. Additional comparison of the absorption measurement with the photographic documentation has enabled the identification of a part of the characteristic frequencies in the spectrum of $|\mathrm{CSDF}|$, allowing them to bind with the flow and the size of the smaller structures produced for this type of flow.
The conducted experiments showed that for the flow in horizontal pipelines with diameters comparable to the size of the crystals of scintillation probes presented the discussed system can be competitive with tomographic methods. The use of mathematical methods for signal analysis in both time and frequency domains allows to determine a larger number of flow parameters than which was measured before with gamma ray absorption.

In order to confirm the suitability of the considerations described in the article, further tests of the method of absorption of gamma radiation to other types of flow and conduits positioned at different angles will be carried out. In addition, the authors plan to conduct simulation experiments, which would allow to optimize the geometry of the measurement and calibration system without the need to conduct the experiment.

\section{Funding sources}

This publication is funded by AGH University of Science and Technology (No 11.11.140.645).

\section{References}

1. M. Bottin, et al, Int. J. Multiphas. Flow 60, 161-179 (2014)

2. T. Xue, L. Qu, Z. Cao, T. Zhang, Flow Meas. Instrum. 27, 29-36 (2012)

3. M.R. Rzasa, Nucl. Eng. Des. 239, 699-707 (2009)

4. Z. Yu, B. Qincheng, H. Richa, Appl. Therm. Eng. 60, 398-410 (2013)

5. V. Mosorov, Meas. Sci. Technol. 17, 753-760 (2006)

6. M. Zych, et al, Flow Meas. Instrum. 35, 11-15 (2014)

7. R. Hanus, L. Petryka, M. Zych, Flow Meas. Instrum. 40, 58-63 (2014)

8. L. Petryka, M. Zych, R. Murzyn, Nukleonika 50, 43-46 (2005)

9. R. Hanus, Int. J. Multiphas. Flow 72, 210-217 (2015)

10. M. Zych, R. Hanus, L. Petryka, MATEC WoC 18, 02004 (2014)

11. M. Zych, et al., JPCS (to be published)

12. J.S. Bendat, A.G. Piersol, Random data - analysis and measurement procedures (John Wiley, New York, 2010)

13. W.Q. Yang, M.S. Beck, Flow Meas. Instrum. 8, 7784 (1998)

14. M.S. Beck, A. Plaskowski, Cross correlation flowmeters - their design and application (Adam Hilger, Bristol, 1987)

15. Guide to the expression of uncertainly in measurement. (International Organisation for Standardisation, 1995) 